\title{
Multimode Network Analysis of Planar Transmission Lines
}

\author{
Marco Guglielmi and Alejandro Alvarez Melcon
}

\begin{abstract}
An alternative approach for the full wave analysis of single or coupled planar transmission lines is presented. Following the proposed approach, a multiple (or single) transmission line structure is viewed as series of staked (or single) transverse discontinuities in a parallel plate waveguide. As a consequence, both top and side coupled structures can be studied. Each individual discontinuity is described in terms of a multimode equivalent network representation. The complete cross section of the planar transmission line structure is then described in terms of a transverse equivalent network and a simple transverse resonance technique is then used to obtain the dispersion behavior, the modal field of the structure, and the characteristic impedances. The multimode equivalent network representations of the individual discontinuities used in this paper have been derived elsewhere and are given in terms of impedance or admittance matrices that are essentially independent from frequency. As a result, they need to be computed only once for each given geometry thus leading to codes that are computationally very efficient.
\end{abstract}

\section{INTRODUCTION}

$\mathbf{T}$ THE BASIC transmission line geometries treated in this paper are shown in Fig. 1. The analysis of this type of structures is a subject that has always attracted much attention, and many different approaches have been reported in the technical literature [1]. Early contributions are generally based on quasistatic assumptions and are computationally very efficient but can only be used to describe the dominant mode of the structure. More recent contributions are based on mode-matching approaches, so that higher order modes effects can also be accounted for ([2]-[4], for instance), however, the resulting codes can be much less efficient numerically, especially if multiple-line structures are treated. In this paper we present an alternative approach for the analysis of single and coupled planar transmission lines that is at the same time full-wave and very efficient from a computational point of view.

Following the proposed approach, a transmission line structure is viewed as a number of stacked transverse discontinuities in a parallel-plate waveguide. Each discontinuity is characterized in terms of a multimode equivalent network, so that a transverse equivalent network representation of the complete planar transmission line structure can be easily obtained. After that, a simple transverse resonance procedure is used to obtain the dispersion diagram of the structure under investigation.

Manuscript received February 22, 1995; revised August 1, 1995.

The authors are with the European Space Research and Technology Centre (ESTEC), Noordwijk, The Netherlands.

IEEE Log Number 9414842.
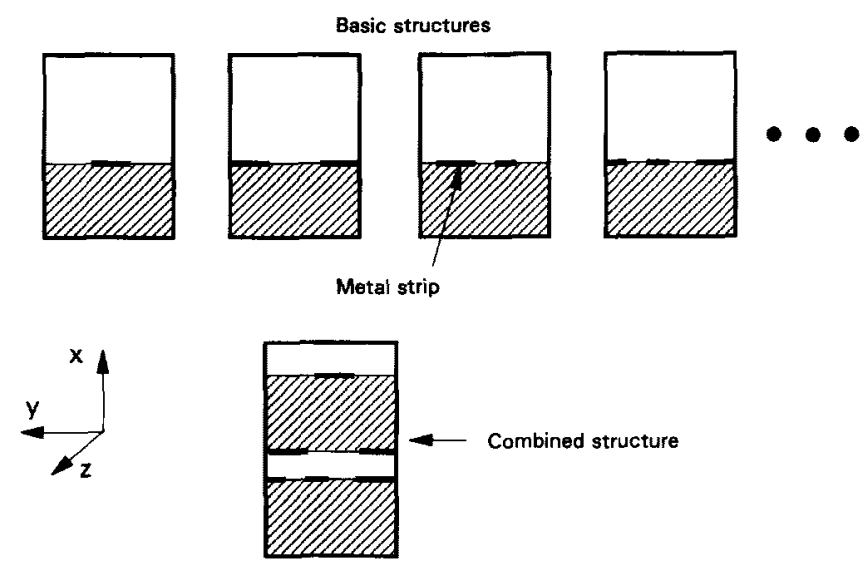

Fig. 1. Basic planner transmission line structures investigated in this paper.

The basic discontinuities involved are the single and double metal strips, the single and double apertures (referred to as the metallic discontinuities), and the air-dielectric interface (Fig. 2). Any staked combination of the basic discontinuities can be easily treated as well. The key feature of this approach is that the multimode characterization of each individual constituent metallic discontinuity is obtained in terms of an impedance (or admittance) matrix that is effectively independent from frequency and absolute dimensions [5]. The frequency dependence is introduced in terms of simple factors, and by the lengths of transmission line that represent the modes of the parallel-plate waveguide in the transverse equivalent networks. This feature greatly reduces the time required for the frequency dependent computations since the characterization of the discontinuities is done only once for each given structure directly in terms of impedance (or admittance) matrix. For each point in frequency, one only needs to solve one linear system, even for complex structures involving more than one line.

One additional important feature of the procedure described in this paper, is the particular decomposition of the basic discontinuity problem to account for the air dielectric interface. In general, the problem of a metal strip at a dielectric interface is a vector problem since such discontinuity couples $\mathrm{E}$ and $\mathrm{H}$ modes to each other. It can be shown, however, that if the transverse discontinuity involves only metal strips (or apertures) that are uniform in a transverse direction, the coupling is basically due to the change in dielectric and not to the metallic discontinuity [9]. The result of this is that the metal strip (or aperture) problem can be treated separately as a scalar problem, the coupling due to the dielectric can be reintroduced later at the equivalent network level. The 


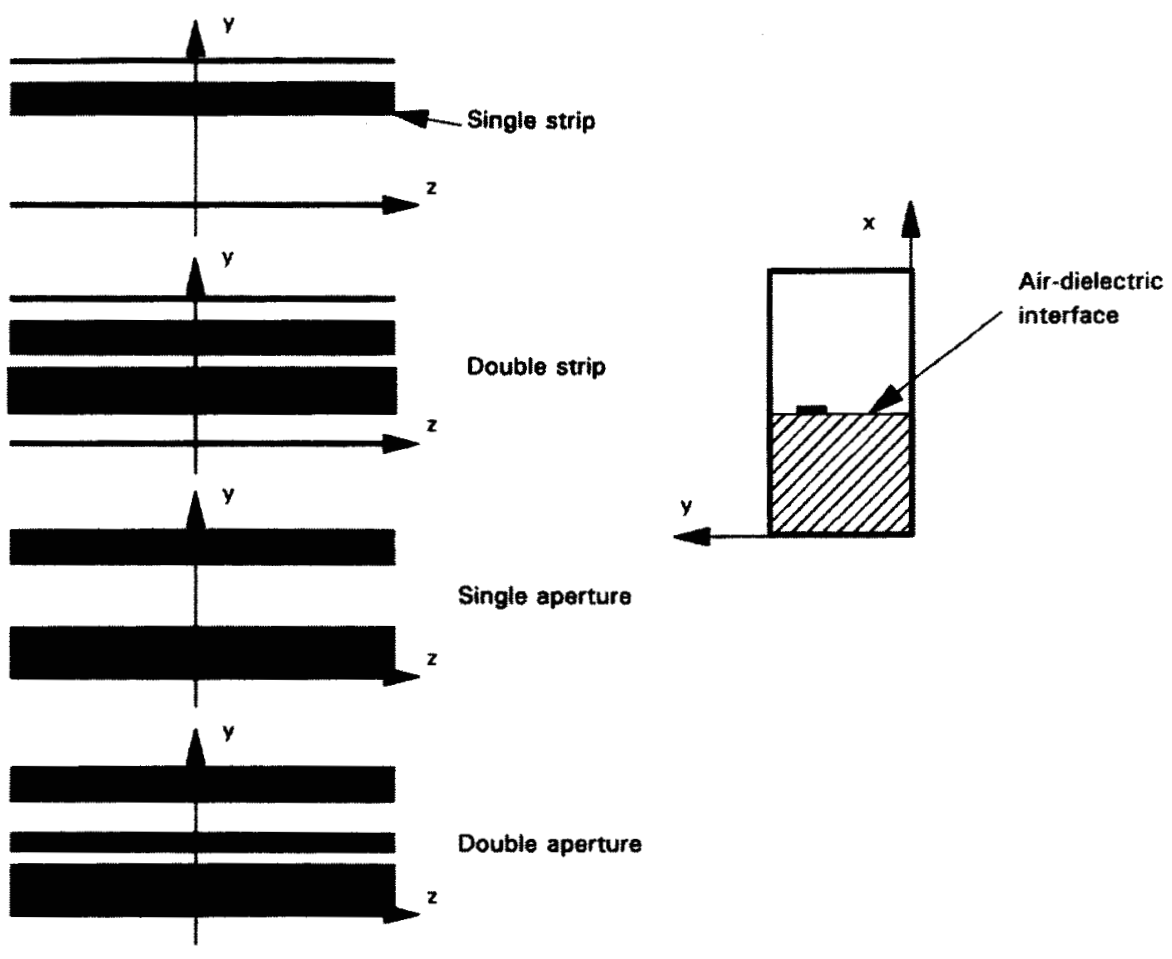

Fig. 2. Basic constituent discontinuities of the transmission lines in Fig. 1.

coupling between $\mathrm{E}$ and $\mathrm{H}$ modes at the dielectric interface turns out to involve only modes with the same wavenumbers, and it can be described in terms of a very simple network (a single transformer) [10].

The original problem can, therefore, be viewed as two separate discontinuity problems that can be solved independently from each other: a metallic discontinuity and a dielectric interface. The original structure is then simply recovered by letting the distance between the two discontinuities go to zero in the equivalent network representation. This concept was already partially outlined in [9] for a single mode situation. The authors of [9], however, could not use the limiting process because the equivalent networks at their disposal could only account for fundamental-mode interactions.

In this paper we describe in detail the procedure for obtaining the transverse equivalent network representation for a variety of single and coupled planar transmission lines. In addition we indicate how, given the form of the multimode equivalent networks, one can develop codes that are particularly efficient from a numerical point of view. Several examples of specific applications are also discussed, and comparisons with measurements and already published data are performed in order to validate the results obtained. As a further application, the full modal spectrum that is supported by a boxed microstrip line is investigated, clarifying some aspects not previously discussed (to the authors' knowledge) in the technical literature.

\section{BASIC DisCONTINUITIES}

The basic discontinuities used in this paper are the single and double metallic strip, the single and double aperture in a parallel plate waveguide, and the air-dielectric interface, as shown in Fig. 2. The modal expansions that we employed for deriving the multimode equivalent networks of the individual discontinuities involve the hybrid $H$-Type and $E$-Type modes and the propagation direction is assumed to be the $x$ direction of Fig. 2. Our final goal is to obtain the wavenumber in the $z$ direction for the class of structures being investigated. It turns out, however, that the network representations for the case $k_{z} \neq 0$ can be simply derived from the one for $k_{z}=0$ by substituting $k_{o}^{2}$ with $k_{o}^{2}-k_{z}^{2}$ everywhere in the network representation [9]. Following this viewpoint, the problem becomes significantly simpler since the hybrid modes reduce to the standard $\mathrm{TE}_{x}$ and $\mathrm{TM}_{x}$ of the parallel plate waveguide (hybrid modes at normal incidence).

\section{A. Metallic Discontinuities}

For the problem at hand, we have four basic discontinuities and we need two networks for each discontinuity, one under $H$-Type and the other under $E$-Type excitation, respectively. The detailed description of the rigorous solution of these problems can be found in [5], for the zero-thickness case, and are not reported here. What is important to note in this context is that the general form of the multimode network representation for all discontinuities is always the one shown in Fig. 3, for both $H$-Type or $E$-Type excitation (the same network representations is valid also for the case in which an arbitrary number of strips or apertures were used).

Furthermore, the expressions for the generic element of the multimode impedance or admittance coupling matrices essentially do not depend on frequency or absolute dimensions. As a consequence, one can generate a finite set of coupling matrix elements corresponding to a discrete set of geometries by using the full formulation and store the results obtained 


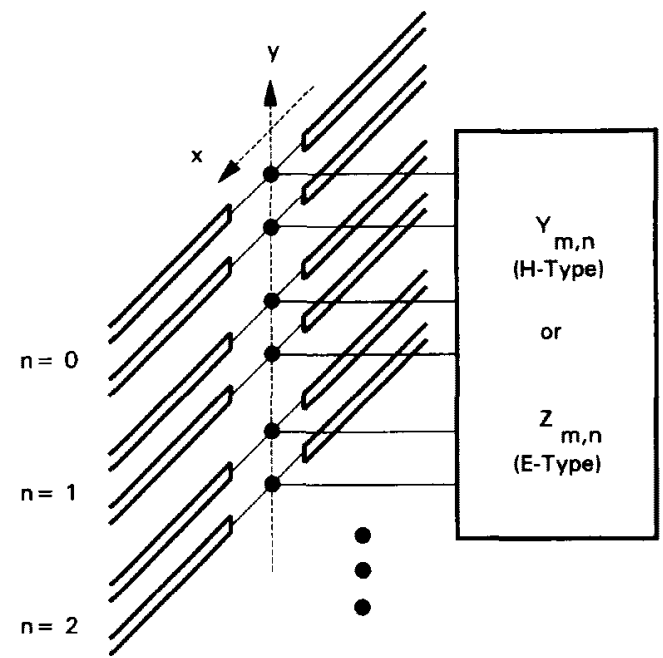

Fig. 3. Typical multimode equivalent network representation for the metallic discontinuities in Fig. 2 for both $H$-Type and $E$-Type incident modes.

in appropriate files. Any other coupling matrix relevant to a specific geometry can then be obtained very rapidly using an interpolation procedure without any additional complex computations.

The problem under discussion involves zero-thickness metal strips, as already mentioned. The procedure, however, is completely general so that structures with finite thickness can be treated as well. The only difference is that the constituent discontinuities would become the ones described in [6] rather than the ones used in this paper. For the sake of space, however, only the zero-thickness case is treated here.

\section{B. Dielectric Interface}

The problem of the coupling between hybrid modes at an air dielectric interface was first studied in network terms in [11], in the context of radial transmission lines. The same concept was further extended to a parallel-plate waveguide airdielectric interface in [10], in the context of leaky-wave non radiative dielectric waveguide antennas. A simple equivalent network representation was, in fact, derived for the coupling of the first higher order hybrid modes for the structure in Fig. 1 in terms of a single transformer. The procedure followed in [10] for the development of the equivalent network is based on the imposition of the continuity of the transverse field components and clearly indicates that coupling can only occur between modes with the same transverse wavenumbers. Although the network was developed for single mode applications, the same procedure can be used in a multimode situation. Extending the results given in [10] to the case of interest, we can easily obtain the equivalent network shown in Fig. 4, where the turn ratio of the transformer is simply given by

$$
N_{n}=\beta_{n}\left(\frac{n \pi}{b}\right) \frac{k_{o}^{2}\left(\epsilon_{r}-1\right)}{\left(k_{o}^{2}-\beta_{n}^{2}\right)\left(k_{o}^{2} \epsilon_{r}-\beta_{n}^{2}\right)} .
$$

\section{TRANSVERSE EQuivalent Networks AND APPLICATIONS}

We have, at this point, all of the key elements to build the transverse equivalent network representation for a large variety

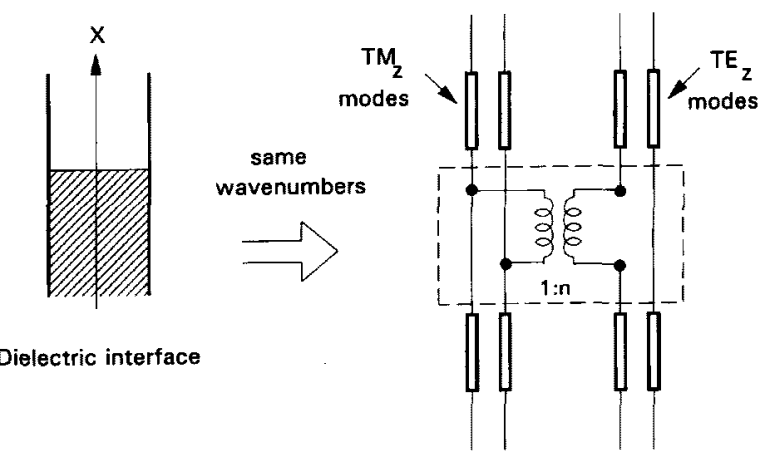

Fig. 4. Equivalent network representation of the air-dielectric interface in Fig. 1.

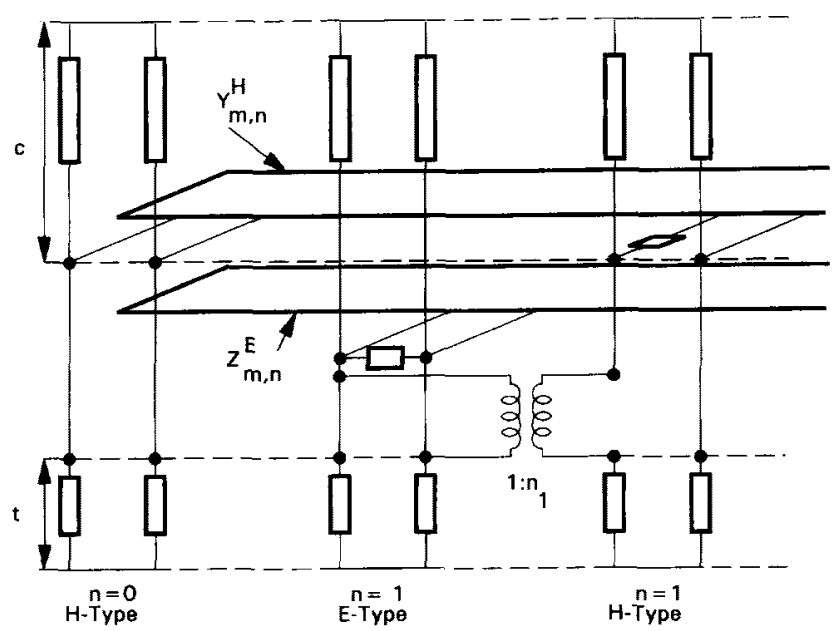

Fig. 5. Complete equivalent network representation of the boxed microstrip in Fig. 1.

of planar transmission line structures, and we now discuss in detail the application of the theory developed to a number of specific cases.

\section{A. Boxed Microstrip}

The complete transverse equivalent network representation for the boxed microstrip in Fig. 1 is shown in Fig. 5. Only three modes are included explicitly in the Fig. but more can be easily added. Starting from the top, the lengths of transmission line represent the modes of the air-filled parallelplate waveguide including the effect of the top cover (short circuit termination). At the location of the metal strip we observe two sets of multimode coupling matrices. They represent the higher order mode couplings that takes places at the discontinuity independently for $H$ - and $E$-Type modes. The coupling between $H$ - and $E$-Type is then accounted for by the transformers involving only pairs of modes with the same wavenumbers. The additional length of transmission line represent the modes of the dielectric-filled parallel plate waveguide, with the short circuit termination to represent the bottom ground plane. The multimode coupling matrices for the strip discontinuity have been derived for the case in which air is present on both sides of the strip and a fictitious distance 


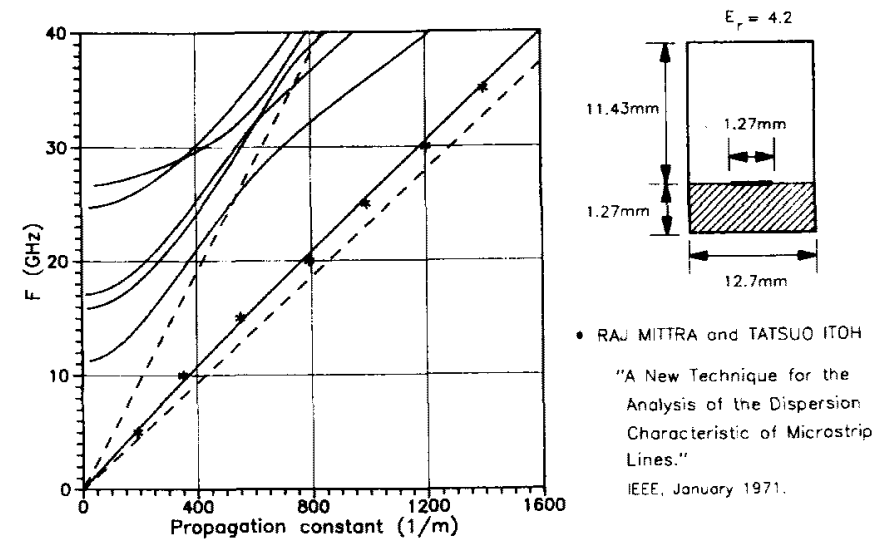

Fig. 6. Dispersion diagram for a boxed microstrip. The star indicate the result presented in [12]

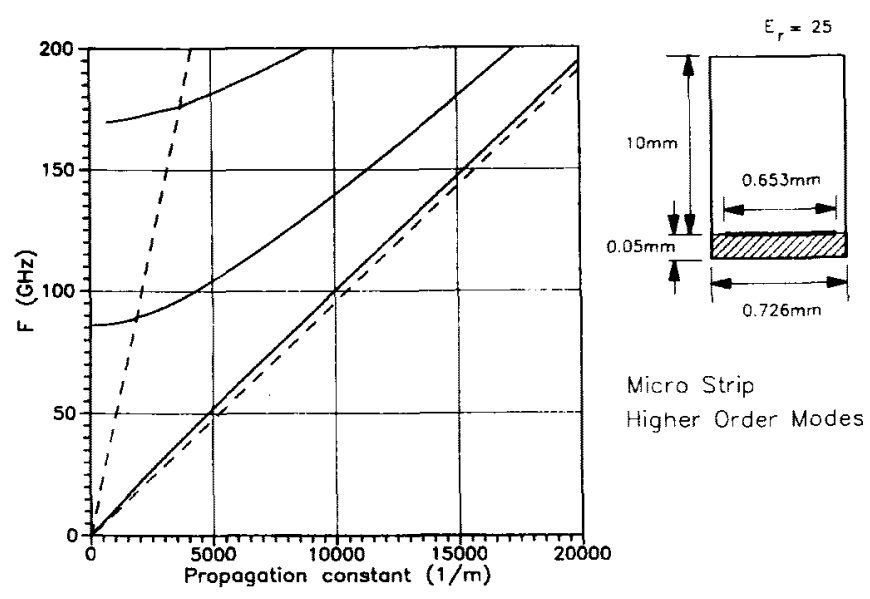

Fig. 7. Same as Fig. 6, microstrip modes.

has then been introduced to separate the metal strip from the air-dielectric interface. The network in Fig. 5 is obtained by letting this fictitious distance go to zero.

Once the transverse equivalent network representation is available, a simple transverse resonance procedure can directly give the dispersion diagram of the modes of the structure. Fig. 6 shows the results obtained for the dispersion diagram of a structure with the same physical parameters as the one in [12]. As we can see the agreement for the lowest mode is very good. Note that the dashed lines in Fig. 6 (and also in Figs. 7-9) represent the dispersion diagrams of two plane waves, one in vacuum (upper curve), and the other in the dielectric medium (lower curve), respectively.

As an application of the theory developed, we have carried out a detailed study of the boxed microstrip in order to clarify the nature its modal spectrum. This structure has been studied by many authors in great detail but, to our knowledge, only with respect to the fundamental mode while all other modes have been included in the general category of "higher order modes." We actually find that there are three types of higher order modes, if propagation is assumed in the $z$ direction of Fig. 1. These modes are:

1) Microstrip modes.
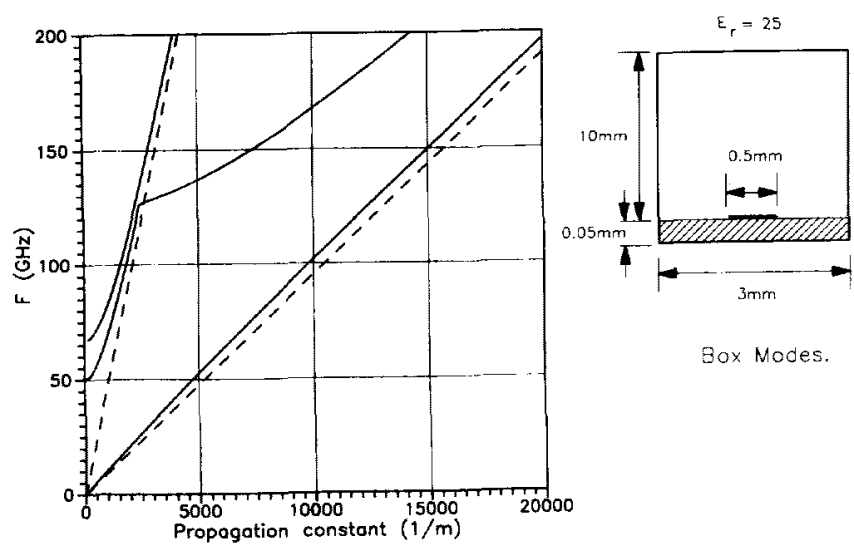

Fig. 8. Same as Fig. 6, box modes.

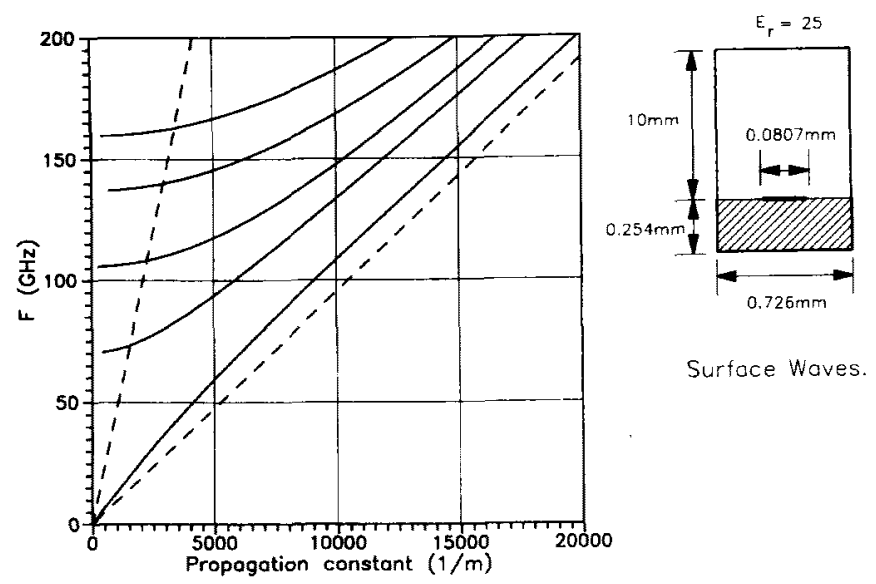

Fig. 9. Same as Fig. 7, surface wave modes.

2) Box modes.

3) Surface wave modes.

The microstrip higher-order modes have been defined as the solutions of the transverse resonance equation that can be obtained by modifying the boxed microstrip so that this partial modal set becomes the lowest set of modes. The modification consists of reducing the height of the dielectric layer while increasing the width of the microstrip. Fig. 7 shows the dimensions of the structure and its dispersion diagrams. In a normal situation (50 ohm line, 1 to $20 \mathrm{GHz}$ ) the first higher order mode of this set would begin propagating far outside the frequency range of interest.

The second set of higher-order modes has been denominated "box modes" because, at low frequency, they essentially represent energy propagation in the air space above the microstrip (Fig. 8). In fact, the dispersion diagram in this region is very similar the one of an empty waveguide of dimensions identical to the ones of the box. As frequency increases, the energy is eventually confined inside the dielectric layer. The dispersion diagram of the first higher-order mode in this region becomes, in fact, almost identical to the one of a dielectric filled metallic waveguide. As a result of this change, the dispersion diagram in Fig. 8 exhibits a very sharp variation when crossing the dashed line corresponding to plane-wave 

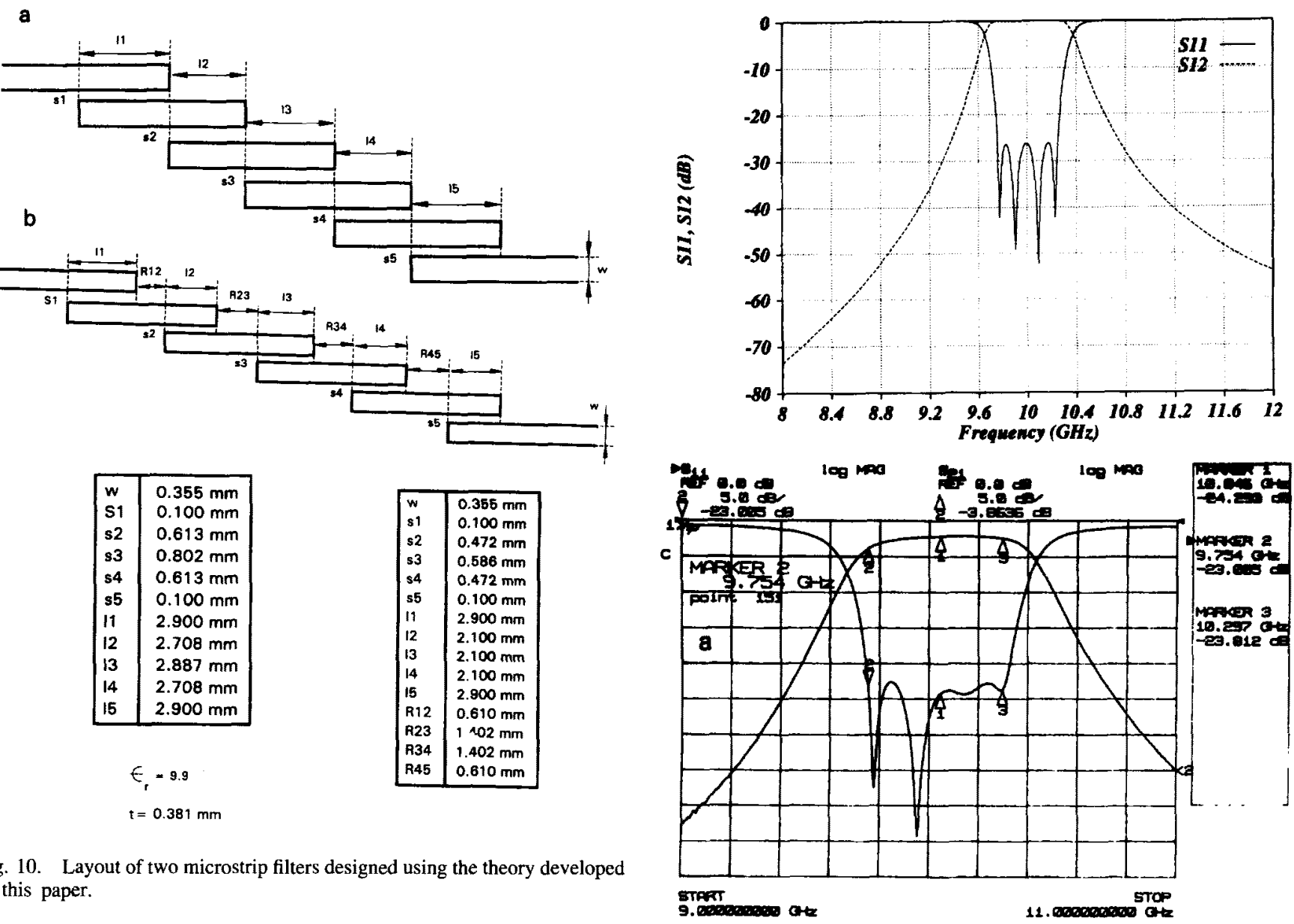

Fig. 10. Layout of two microstrip filters designed using the theory developed in this paper.

propagation in vacuum. To put in evidence this set of modes, we have reduced both the width of the microstrip and the thickness of the dielectric layer, while enlarging the lateral width of the box.

Finally, the third set of modes, namely the "surface wave" modes have been obtained by reducing the width of the metal strip and the lateral extent of the box while increasing the thickness of the dielectric layer. Fig. 9 shows the dispersion diagrams obtained clearly indicating that the energy, for all modes, appears to be confined in the dielectric layer, even at low frequencies. The surface wave modes are the higher order modes that are normally more important for the geometries commonly used. It is interesting to note that these modes exist, although slightly modified, even if the microstrip is completely removed.

The typical computation effort required to obtain any of the dispersion diagrams discussed can be essentially divided into two parts. The first part is for the computation of the coupling matrix elements, the second part is for the solution of the transverse resonance equation. Using an IBM RISC workstation, the first step requires about one minute, while the second requires about 0.05 seconds per frequency point, using a total of 71 modes in the equivalent networks. It is important to note that the coupling matrix calculations are carried out only once for each given structure and only the transverse resonance equation needs to be solved for each point in frequency. The time required for each dispersion curve

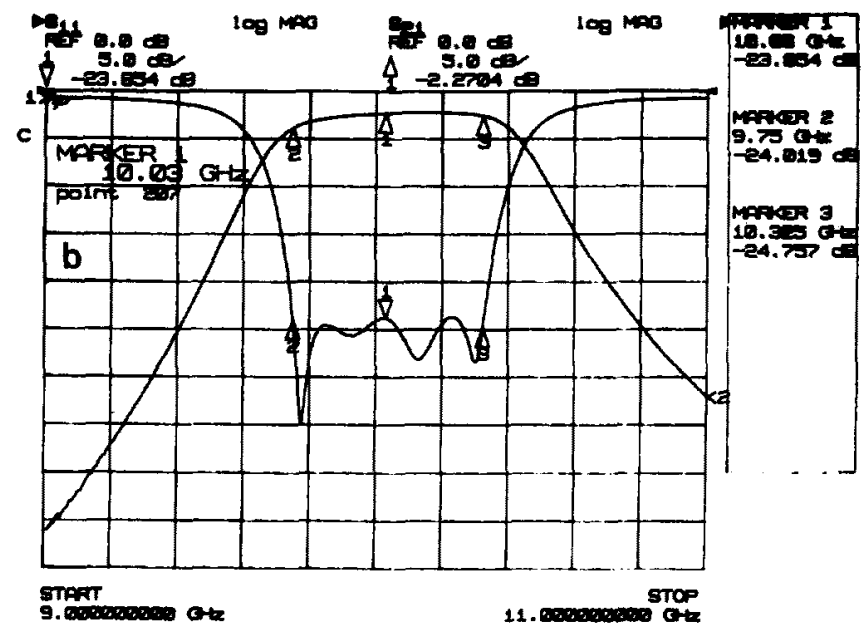

Fig. 11. Measured versus simulated performance of the filters in Fig. 10.

calculation with 200 points in frequency is therefore about 10 seconds.

\section{B. Microstrip filters}

As a further example of application, we presents now the results obtained for two microstrip filters based on coupled line geometries. Fig. 10 shows the actual layout and dimensions of the filters, while Fig. 11 shows the measured versus simulated results (both filters have essentially the same ideal response 
so that a single ideal curve is presented in Fig. 11). The filters have been dimensioned using the procedure described in [13], together with the networks developed in this paper for the characterization of the coupled line sections. As we can see the agreement between simulation and measurement is excellent.

\section{CONCLUSION}

In this paper we have described an alternative procedure for the analysis of single and coupled planar transmission line structures. The method described is based on the decomposition of the global structure into constituent discontinuities in a parallel plate waveguide environment. Each of the constituent discontinuities is studied separately obtaining individual multimode equivalent network representations. Once each discontinuity is known, a global equivalent network representation is developed and a simple transverse resonance technique is then used to extract the electrical parameters of interest. As application example, the full modal spectrum of the boxed microstrip is discussed and several types of higher order modes are identified. Finally, measured and simulated results are presented for two microwave filters designed using the software developed indicating very good agreement between theory and measurements.

\section{REFERENCES}

[1] T. Itoh, Ed., Planar Transmission Line Structures. New York: IEEE Press, 1987.

[2] R. R. Mansour and R. H. Macphie, "A unified hybrid-mode analysis for planar transmission lines with multilayer isotropic/anisotropic substrates," IEEE Trans. Microwave Theory Tech., vol. MTT-35, no. 12, pp 1382-1391, Dec. 1987.

[3] J. Bornemann, "A scattering-type transverse resonance technique for the calculation of M(MIC) transmission line characteristics," IEEE Trans. Microwave Theory Tech., vol. 39, no. 12, pp. 2083-2088, Dec. 1991.

[4] Z. Ma, E. Yamashita, and S. Xu, "Hybrid-mode analysis of planar transmission lines with arbitrary metallization crossection," IEEE Trans. Microwave Theory Tech., vol. 41, no. 3, pp. 491-497, March 1993.

[5] M. Guglielmi and G. Gheri, "Multimode network representation of multiple inductive and capacitive obstacles in parallel plate waveguides," IEEE Trans. Microwave Theory Tech., vol. 42, no. 6, pp. 1046-1051, June 1994.

[6] __ "Multimode equivalent network representation of inductive and capacitive multiple posts," IEE Proc. Microwave Antennas Propag., vol. 42, no. 1, pp. 41-46, Feb. 1995.

[7] J. C. Rautio and R. F. Harrington, "A time-harmonic electromagnetic analysis of shielded microstrip circuits," IEEE Trans. Microwave Theory Tech., vol. MTT-35, no. 8, pp. 726-730, Aug. 1987.

[8] L. P. Dunleavy and P. B. Katehi, "A generalized method for analyzing shielded thin microstrip discontinuities," IEEE Trans. Microwave Theon Tech., vol. 36, no. 12, pp. 1758-1766, Dec. 1988.

[9] H. M. Altschuler and L. O. Goldstone, "On network representation of certain obstacles in waveguide regions," IRE Trans. Microwave Theory Tech., pp. 213-221, Apr. 1959.
[10] A. Sanchez and A. A. Oliner, "A new leaky waveguide for millimeter waves using nonradiative dielectric (NRD) waveguide-Part I: Accurate theory," IEEE Trans. Microwave Theory Tech., vol. MTT-35, no. 8, pp. 737-747, Aug. 1987.

[11] P. J. B. Clarricoats and A. A. Oliner, "Transverse-network representation for inhomogeneously filled circular waveguides," Proc. Inst. Elec. Eng., vol. 112, 1965, pp. 883-894.

[12] R. Mittra and T. Itoh, "A new technique for the analysis of the dispersion characteristics of microstrip lines," IEEE Trans. Microwave Theory Tech., pp. 54-63, Jan. 1971.

[13] M. Guglielmi, "A simple CAD procedure for microwave filters and multiplexers," IEEE Trans. Microwave Theory Tech., vol. 42, no. 7, pp. 1347-1352, July 1994.

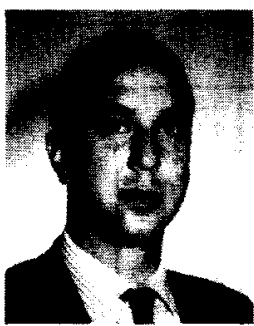

Marco Guglielmi was born in Rome, Italy, on December 17, 1954. He received the "Laurea in Ingegneria Elettronica" degree in 1979 from the University of Rome "La Sapienza," Rome, Italy, where in 1980 he also attended the "Scuola di Specializzazione in Elettromagnetismo Applicato." In 1981 he was awarded a Fulbright Scholarship in Rome, Italy, and an HISP (Halsey International Scholarship Program) from the University of Bridgeport, Bridgeport, CT, USA, where in 1982 he received the M.S. degree in electrical engineering. In 1986 he received the Ph.D. degree in electrophysic from the Polytechnic University, Brooklyn, NY, USA.

From 1984 to 1986 he was Academic Associate at the Polytechnic University, and from 1986 to 1988 he was Assistant Professor at the same institution. From 1988 to 1989 he was Assistant Professor at the New Jersey Institute of Technology, Newark, NJ USA. In 1989 he joined the RF System Division of the European Space Research and Technology Centre, Noordwijk, The Netherlands, where he is currently involved in the development of passive microwave components for space application. His professional interests include the areas of solid-state devices and circuits, periodic structures, phased arrays and millimeter-wave antennas, network representation of waveguide discontinuities and microwave filtering structures.

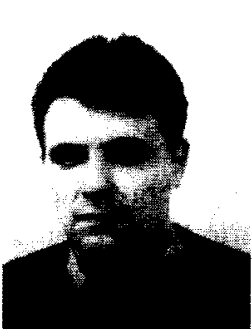

Alejandro Alvarez Melcon was born in Madrid Spain, in 1965. He received the "Ingeniero de Telecomunicaciones" degree from the Polytechnic University of Madrid (UPM) in 1991.

In 1988 he joined the Signal, Systems and Radiocommunications Department of UPM, as a Research Student, where he was involved in the design, testing, and measurement of broadband spiral antennas for EMS equipment. From 1991 to 1994 he was with the Radio Frequency Systems Division of the European Space Agency in Noordwijk, The Netherlands, where he was involved in the development of analytical and numerical tools for the study of waveguide discontinuities, planar transmission lines, and microwave filters. In 1994 he joined the Ecole Polytechnique Federale de Lausanne, Lausanne, Switzerland, where he is currently working toward the $\mathrm{Ph} . \mathrm{D}$. degree in applied electromagnetism. 